\title{
The reliability of toe systolic pressure and the toe brachial index in patients with diabetes
}

\author{
Mary Romanos ${ }^{1 *}$, Anita Raspovic ${ }^{1}$, Byron Perrin ${ }^{2}$ \\ From Australasian Podiatry Council Conference 2011 \\ Melbourne, Australia. 26-29 April 2011
}

\section{Background}

The ankle brachial index is a useful clinical test for establishing blood supply to the foot. However, there are limitations to this method when conducted on people with diabetes. As an alternative to the ankle brachial index, measuring toe systolic pressures and the toe brachial index have been recommended to assess the arterial blood supply to the foot. This study aimed to determine the intra and inter-rater reliability of the measurement of toe systolic pressure and the toe brachial index in patients with diabetes using a manual sphygmomanometer and photoplethysmography unit.

\section{Methods}

This was a repeated measures, reliability study. Three raters measured toe systolic pressure and the toe brachial index in thirty participants with diabetes. Measurement sessions occurred on two occasions, one week apart, using a manual photoplethysmography unit (Hadeco Smartdop 45) and a standardised measurement protocol. Intra-class correlation coefficients and 95\% limits of agreement (LOA) were calculated.

\section{Results}

The mean intra-class correlation for intra-rater reliability for toe systolic pressures was 0.87 (95\% LOA: -25.97 to $26.06 \mathrm{mmHg}$ ) and the mean intra-class correlation for Toe Brachial Indices was 0.75 (95\% LOA: -0.22 to $0.28)$. The intra-class correlation for inter-rater reliability was 0.88 for toe systolic pressures (95\% LOA: -22.91 to $29.17 \mathrm{mmHg}$ ) and 0.77 for toe brachial indices $(95 \%$ LOA: -0.21 to 0.22 ).

\footnotetext{
* Correspondence: mary_romanos@hotmail.com ${ }^{1}$ Department of Podiatry and Musculoskeletal Research Centre, Faculty of Health Sciences, La Trobe University, Bundoora, Victoria, 3086, Australia Full list of author information is available at the end of the article
}

\section{Conclusions}

Despite the reasonable intra-class correlation results, the range of error was broad. This potentially clinically significant margin of error raises questions about the reliability of using a manual sphygmomanometer and photoplethysmography to measure toe systolic pressure and toe brachial index. When assessing patients with peripheral arterial occlusive disease, it is important to consider all other non-invasive vascular assessment options.

\section{Author details}

'Department of Podiatry and Musculoskeletal Research Centre, Faculty of Health Sciences, La Trobe University, Bundoora, Victoria, 3086, Australia. ${ }^{2}$ La Trobe Rural Health School and Musculoskeletal Research Centre, Faculty of Health Sciences, La Trobe University, Bendigo, Victoria, 3552, Australia.

Published: 20 May 2011

doi:10.1186/1757-1146-4-S1-P52

Cite this article as: Romanos et al:: The reliability of toe systolic pressure and the toe brachial index in patients with diabetes. Journal of Foot and Ankle Research 2011 4(Suppl 1):P52.

Submit your next manuscript to BioMed Central and take full advantage of:

- Convenient online submission

- Thorough peer review

- No space constraints or color figure charges

- Immediate publication on acceptance

- Inclusion in PubMed, CAS, Scopus and Google Scholar

- Research which is freely available for redistribution 